\title{
Quasi-elastic reactions : an interplay of reaction dynamics and nuclear structure
}

S. Szilner ${ }^{1, a}$, T. Mijatović ${ }^{1}$, L. Corradi ${ }^{2}$, G. Pollarolo ${ }^{3}$, F. Haas ${ }^{4}$, S. Courtin ${ }^{4}$, E. Farnea ${ }^{5}$, E. Fioretto ${ }^{2}$, A. Gadea $^{2}{ }^{6}$, A. Goasduff $^{4}$, D. Jelavić-Malenica ${ }^{1}$, S. Lunardi ${ }^{5}$, N. Mărginean ${ }^{7}$, G. Montagnoli ${ }^{5}$, D. Montanari ${ }^{5}$, F. Recchia ${ }^{5}$, E. Sahin $^{2}$, F. Scarlassara ${ }^{5}$, N. Soić ${ }^{1}$, A. M. Stefanini ${ }^{2}$, C. A. Ur ${ }^{5}$, and J. J. Valiente-Dobón ${ }^{2}$

1 Ruđer Bošković Institute, Zagreb, Croatia

2 INFN - Laboratori Nazionali di Legnaro, Legnaro, Italy

3 INFN and Università di Torino, Italy

4 IPHC, CNRS/IN2P3 and Université de Strasbourg, Strasbourg, France

5 INFN and Università di Padova, Padova, Italy

6 IFIC, CSIC-Universidad de Valencia, Valencia, Spain

7 Horia Hulubei National Institute of Physics and Nuclear Engineering, Bucharest, Romania

\begin{abstract}
Multinucleon transfer reactions have been investigated in ${ }^{40} \mathrm{Ar}+{ }^{208} \mathrm{~Pb}$ with the Prisma+Clara set-up. The experimental differential cross sections of different neutron transfer channels have been obtained at three different angular settings taking into account the transmission through the spectrometer. The experimental yields of the excited states have been determined via particle- $\gamma$ coincidences. In odd Ar isotopes, we reported a signif cant population of $11 / 2^{-}$states, reached via neutron transfer. Their structure matches a stretched conf guration of the valence neutron coupled to vibration quanta.
\end{abstract}

\section{Introduction}

A signif cant amount of experimental data on heavy ion transfer reactions collected in the last decade has been shown to be quantitatively described in a reaction model which include elementary degrees of freedom, surface vibrations and single particles [1]. The revival of transfer reaction studies benef ted from the construction of the new generation large solid angle spectrometers based on trajectory reconstruction that reached an unprecedented efficiency and selectivity. The coupling of these spectrometers with large $\gamma$ arrays allowed the identif cation of individual excited states and their population pattern.

The understanding of the reaction mechanism depends strongly on the determination of the absolute cross sections. For the large acceptance magnetic spectrometers it becomes unfeasible to use complex magnetic elements to correct for the ion optical aberrations, thus for the Prisma spectrometer the solution of a simplif ed magnetic element conf guration has been adopted, together with the concept of trajectory reconstruction. To reconstruct the ion path through the spectrometer and to determine the ion transport devoted algorithms were developed. This procedure is very important especially for grazing collisions where a wealth of nuclei are produced in a wide energy and angular range and with cross sections spanning several orders of magnitude.

The coupling of single particle degrees of freedom to nuclear vibration quanta is very important for the under-

\footnotetext{
a e-mail: szilner@irb.hr
}

standing of the transfer strength distribution. These effects, still largely unexplored, are essential for the description of many basic states in the vicinity of closed shells. In this work we study the population of states of particlephonon nature in isotopic chains reached via multiple particle transfer mechanism. In more detail we present some results for the neutron transfer channels populated in the ${ }^{40} \mathrm{Ar}+{ }^{208} \mathrm{~Pb}$ multinucleon transfer reaction.

\section{The measurement and data analysis}

The ${ }^{40}$ Ar beam has been extracted from the ECR ion source of PIAVE, pre-accelerated and injected into the superconductive Linac ALPI with f nal energy of $\mathrm{E}_{\mathrm{lab}}=255 \mathrm{MeV}$. Ions with average intensities of $\sim 7 \mathrm{pnA}$ were impinging onto $\mathrm{a} \simeq 300 \mu \mathrm{g} / \mathrm{cm}^{2}{ }^{208} \mathrm{~Pb}$ target (99.9\% isotopically enriched). Projectile-like fragments have been identif ed with the large solid angle magnetic spectrometer Prisma [2-4], while the coincident $\gamma$ rays were detected with the Clara array [5], situated in the hemisphere opposite to Prisma. The ${ }^{40} \mathrm{Ar}+{ }^{208} \mathrm{~Pb}$ reaction has been measured at three different Prisma angles $\theta_{\mathrm{lab}}=46^{\circ}, 54^{\circ}\left(\approx \theta_{\text {grazing }}\right)$ and $59^{\circ}$ to cover most of the transfer f ux. The normalization for the different measured angles was ensured by a silicon SSBD monitor detector positioned at a forward angle. At the grazing angle, coincident $\gamma$ rays were measured for a longer time to achieve sufficient statistics for detailed studies of the transfer strength distribution over the f nal excited states. 

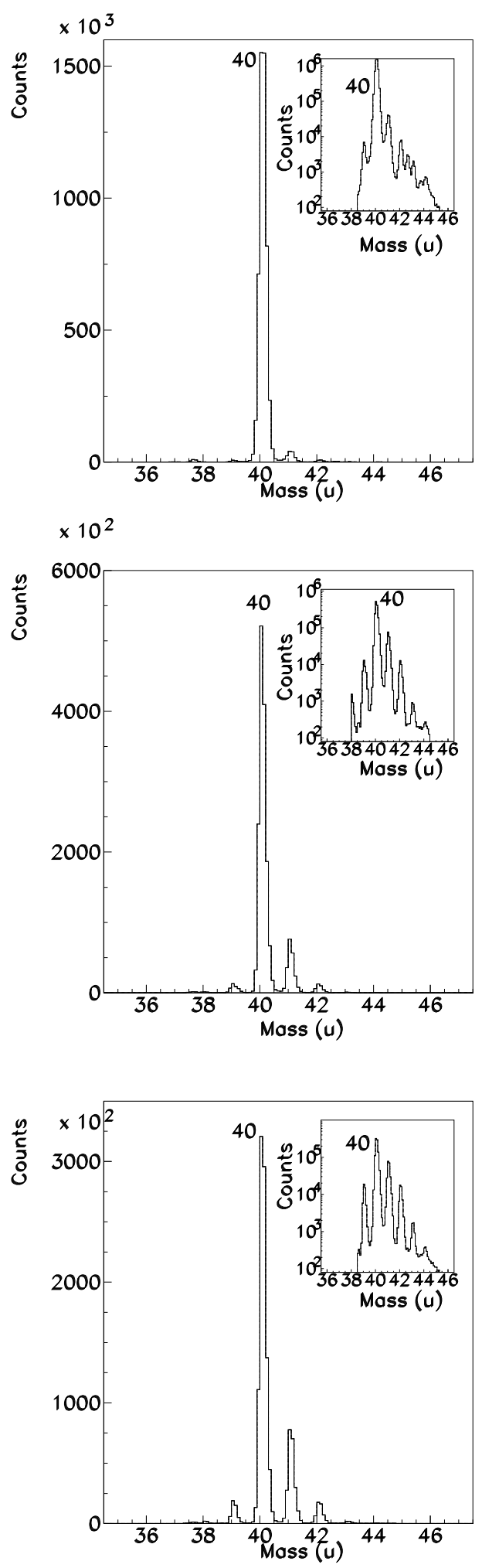

Fig. 1. Mass distributions of argon isotopes populated in ${ }^{40} \mathrm{Ar}+{ }^{208} \mathrm{~Pb}$ at $E_{\text {lab }}=255 \mathrm{MeV}$ and at $\theta_{\mathrm{lab}}=46^{\circ}, 54^{\circ}$ and $59^{\circ}$ (from top to bottom). A mass resolution of $\triangle A / A \sim 1 / 160$ has been obtained. Inserts display the same mass distribution in logarithmic scale. Please notice that the difference in yields between ${ }^{40} \mathrm{Ar}$ and ${ }^{43} \mathrm{Ar}$ at the most forward measured angle is more than 3 orders of magnitude.
An event-by-event reconstruction of the ion trajectory inside the Prisma magnetic elements is done making use of position information from the entrance and focal plane detectors together with time-of-f ight [6]. The nuclear charge has been obtained through the measurement of energy loss and total energy. The quality of the trajectory reconstruction can be appreciated in Fig. 1 which shows the mass distribution of the neutron transfer channels. The precision of the reconstructed vector velocity can be seen also from the Doppler correction of the coincident $\gamma$ rays (see later).

To evaluate cross sections we have studied the response function of the Prisma spectrometer which depends in a complex way on the entrance angles and momenta of the reaction products [7]. In more details, to calculate the response function of the spectrometer we generate a distribution that is uniform in energy and isotropic in angle. The energy range of this distribution corresponds to the momentum acceptance of the spectrometer, while the angular range is taken to be slightly larger than the Prisma entrance area. The distribution is then transported to the focal plane by a ray tracing code [7] with the same magnetic felds set in the experiment. The ray tracing code uses numerical integrators to determine the trajectory of individual rays through the electromagnetic f elds. The ratio between the input uniform $(\mathrm{E}, \theta)$ distribution and the distribution of transported ions through the Prisma at the focal plane allows us to def ne a correction factor. As an example, the projection of the two-dimensional correction matrix as a function of the $\theta_{\text {lab }}$ for ${ }^{41} \mathrm{Ar}$ with different cuts in the kinetic energy is displayed in Fig. 2. The transmission factors through Prisma have been applied as a correction to the experimental differential cross sections. As expected, the major contribution is at the borders of the Prisma angular acceptance, depending strongly on the kinetic energy of the transported ions.

The bell shaped angular distributions of the neutron transfer channels agree well, both in the shape and magnitude, with the predictions of the semiclassical model GRAZ ING [8]. This model calculates the evolution of the reaction by taking into account, besides the relative motion variables, the intrinsic degrees of freedom of projectile and target. These are the surface modes and the single-nucleon transfer channels. The multinucleon transfer channels are described via a multistep mechanism. The relative motion of the system is calculated in a nuclear plus Coulomb f eld. The excitation of the intrinsic degrees of freedom is obtained by employing the well-known form factors for the collective surface vibrations and the one-particle transfer channels.

The theory, also, well describes the total inclusive cross sections for the neutron transfer channels. The dependence of the cross sections on the number of transferred neutrons is very similar to the one observed in other studied systems [1]. The neutron pick-up drops by almost a constant factor for each transfered neutron as an independent mechanism would suggest. In the present case a deeper insight may be achieved by measuring the individual $\gamma$-ray transitions and thus the population of the single excited states. 

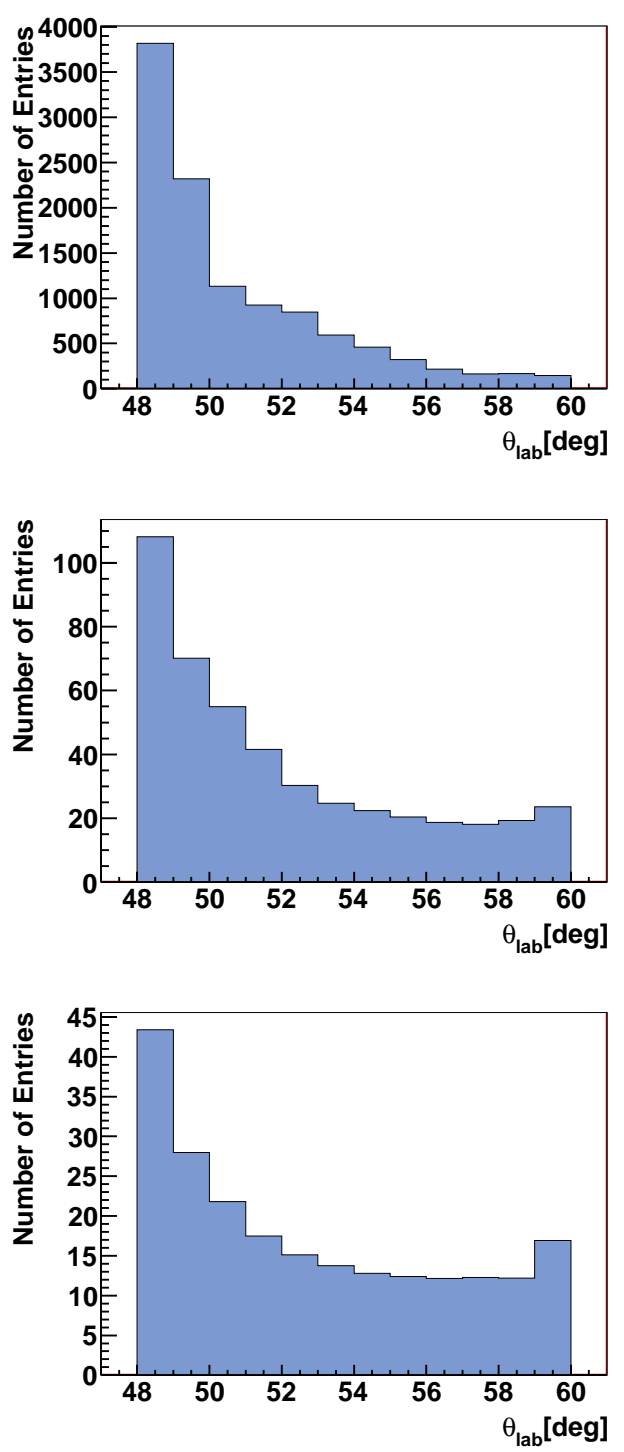

Fig. 2. The projection of the two-dimensional correction matrix for ${ }^{41} \mathrm{Ar}$ with different kinetic energy cuts: $(100 \mathrm{MeV}, 110 \mathrm{MeV})$, $(160 \mathrm{MeV}, 170 \mathrm{MeV})$ and $(240 \mathrm{MeV}, 250 \mathrm{MeV})$ from top to bottom, respectively.

\section{The character of states populated in transfer reactions}

We have interpreted the experimental yields with a model that explicitly treats the internal degrees of freedom of the two ions in terms of elementary modes, surface vibration and single particles. It is through the excitation of these elementary modes that energy and angular momentum are transferred from the relative motion to the intrinsic degrees of freedom and that mass and charge are exchanged between the two partners of the collision. Thus, heavyion collisions provide a suitable tool for the studies of the particle-vibration coupling scheme. Here we concentrate on the neutron transfer channels, and selected $\gamma$ spectra of argon isotopes are plotted in Fig. 3. The fragment- $\gamma$ coincidence obtained from the coupling of Clara with Prisma

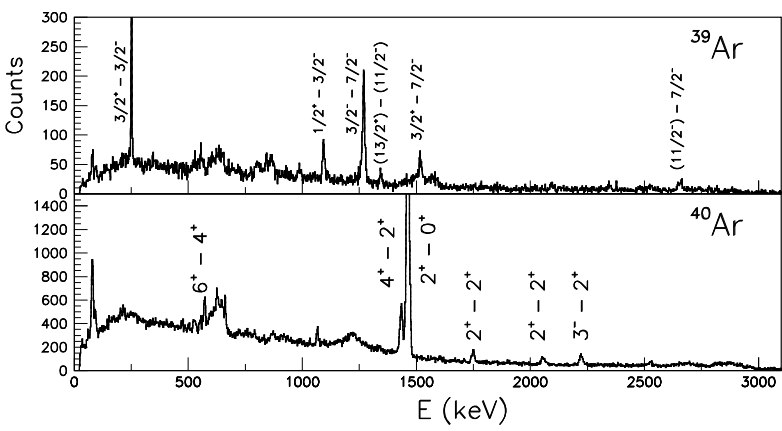

Fig. 3. Doppler corrected $\gamma$-ray spectra for ${ }^{39,40} \mathrm{Ar}$ populated in ${ }^{40} \mathrm{Ar}+{ }^{208} \mathrm{~Pb}$ by detecting the ${ }^{39,40} \mathrm{Ar}$ isotope in Prisma and the coincident $\gamma$ rays in Clara. The strongest transitions are marked in the f gure.

allows us to attribute to each specif c reaction product its characteristic $\gamma$ rays. Since the $\gamma$ rays are emitted in $\mathrm{fight}$ it is mandatory to perform Doppler correction. This is done from the knowledge of the trajectory reconstructed in Prisma, which provides the velocity vector of the emitting nuclei.

The spectra comprise transitions partly from particle states and partly from states that involve combinations of single-particle with a collective boson. In ${ }^{40} \mathrm{Ar}$ the strongest transitions are observed between the yrast states, with the very strong population of $2^{+}$state (we remind that this is the inelastic channel). Besides them, we also observe the non-yrast feeding of the $2^{+}$and $0^{+}$states. The strong population of the yrast states dominates the $+2 n$ channel $\left({ }^{42} \mathrm{Ar}\right)$. In ${ }^{39} \mathrm{Ar}$ the negative parity states, the low lying states with the pronounced single-particle character, are strongly populated. For example the $3 / 2_{1}^{-}$state and its transition to the $7 / 2^{-}$ground state dominates the ${ }^{39} \mathrm{Ar}$ spectra. In addition, we observe, also, a decay of the $11 / 2^{-}$state. The low lying positive parity states have been also populated to some extent. In the more neutron rich argon isotopes, beside the population of the known low-lying states which have been recognized in the spectra, the newly observed transition have been attributed to the yet unknown $11 / 2^{-}$states in ${ }^{41,43} \mathrm{Ar}$. The populated states were compared with the results of the $0 \hbar \omega s d-p f$ large-scale shell-model (SM) calculations $[10,11]$. The recently developed SDPF-U effective interaction, with the valence space of the full $s d$ shell for the protons and the full $p f$ shell for the neutrons, has been used. The energies, spins and parities of newly identif ed states agrees well with the SM calculations.

The $11 / 2^{-}$state can be understood as a coupling of collective boson to single-particle states (i.e. $\mid 2^{+},\left(f_{7 / 2}\right)^{1}>$ giving an $11 / 2^{-}$stretched conf guration). It is expected that the properties of these particle-phonon states are to a large extent determined by the properties of the corresponding phonon states. The comparison of the measured and SM calculated energies for the $2^{+}$and $11 / 2^{-}$states of argon isotopes shows an excellent agreement for all argon isotopes in the $N=20-28$ shell. The behavior of their 
energies displays that the evolution of the collectivity, in the even isotopes $\left(2^{+}\right.$energies) and in the odd isotopes $\left(11 / 2^{-}\right.$energies), is very similar. This further corroborates the particle-phonon character of these $11 / 2^{-}$states. We expect that heavy ion induced transfer reactions populate states of similar character in more neutron rich isotopes $[12,13]$. Argon isotopes with $\mathrm{N} \geq 28$ have been populated in ${ }^{238} \mathrm{U}+{ }^{48} \mathrm{Ca}$ reactions [13], and the populated states in the ${ }^{47} \mathrm{Ar}$ behave similarly to here discussed odd-argon isotopes.

As already mentioned, in the reaction model the excitation and transfer processes are mediated by the well known single-particle form factors for the fermion degrees of freedom and by the collective form factors, for the vibrational modes. The signif cant population of such states, reached via neutron transfer, demonstrates the importance of excitation of the states whose structure can be explained with the same degrees of freedom which are needed in the reaction model, i.e. coupling of the valence neutron to the vibration quanta.

\section{Summary}

In transfer reactions one of the most interesting items is how single particle and more complex degrees of freedom act in the transfer process. Via $\gamma$-particle coincidences it is now experimentally possible to measure the transfer strength to specif c f nal states with high efficiency. To better understand the structure of states which are strongly excited by the multi-nucleon transfer mechanism we have studied the experimental yields of populated states in neutron transfer channels in ${ }^{40} \mathrm{Ar}+{ }^{208} \mathrm{~Pb}$. The individual state yield distribution in the f nal reaction products ref ects a strong interplay between single-particle and collective degrees of freedom that is pertinent to the reaction dynamics.

Acknowledgments: This work was partly supported by the Croatian Ministry of Science, Education and Sports, Grant No. 00981191005-2890, and FP7 project CLUNA.

\section{References}

1. L. Corradi, G. Pollarolo and S. Szilner, J. of Phys. G 36, (2009) 113101, and references therein

2. A.M. Stefanini, L. Corradi, G. Maron, A. Pisent, M. Trotta, A.M. Vinodkumar, S. Beghini, G. Montagnoli, F. Scarlassara, G.F. Segato, A. De Rosa, G. Inglima, D. Pierroutsakou, M. Romoli, M. Sandoli, G. Pollarolo, and A. Latina, Nucl. Phys. A701, (2002) 217c

3. G. Montagnoli, A.M. Stefanini, M. Trotta, S. Beghini, M. Bettini, F. Scarlassara, V. Schiavon, L. Corradi, B.R. Behera, R. Fioretto, A. Gadea, A. Latina, S. Szilner, L. Donà, M. Rigato, N.A. Kondratiev, A. Yu. Chizhov, G. Kniajeva, E.M. Kozulin, I.V. Pokrovskiy, V.M. Voshressensky, and D. Ackermann, Nucl. Instr. and Meth. in Phys. Res. A 547, (2005) 455
4. S. Beghini, L. Corradi, E. Fioretto, A. Gadea, A. Latina, G. Montagnoli, F. Scarlassara, A.M. Stefanini, S. Szilner, M. Trotta, and A.M. Vinodkumar, Nucl. Instr. and Meth. in Phys. Res. A 551, (2005) 364

5. A. Gadea, D.R. Napoli, G. de Angelis, R. Menegazzo, A.M. Stefanini, L. Corradi, M. Axiotis, L. Berti, E. Fioretto, T. Kroell, A. Latina, N. Marginean, G. Maron, T. Martinez, D. Rosso, C. Rusu, N. Toniolo, S. Szilner, M. Trotta, D. Bazzacco, S. Beghini, M. Bellato, F. Brandolini, E. Farnea, R. Isocrate, S.M. Lenzi, S. Lunardi, G. Montagnoli, P. Pavan, C.R. Alvarez, F. Scarlassara, C.A. Ur, N. Blasi, A. Bracco, F. Camera, S. Leoni, B. Million, M. Pignanelli, G. Pollarolo, A. DeRosa, C. Inglima, M. La Commara, G. La Rana, D. Pierroutsakou, M. Romoli, M. Sandoli, P.G. Bizzeti, A.M. Bizzeti-Sona, G. Lo Bianco, C.M. Petrache, A. Zucchiatti, P. Cocconi, B. Quintana, C. Beck, D. Curien, G. Duchene, F. Haas, P. Medina, P. Papka, J. Durell, S.J. Freeman, A. Smith, B. Varley, K. Fayz, V. Pucknell, J. Simpson, W. Gelletly, and P. Regan, Eur. Phys. J. A 20 , (2004) 193

6. S. Szilner, C. A. Ur, L. Corradi, N. Marginean, G. Pollarolo, A. M. Stefanini, S. Beghini, B. R. Behera, E. Fioretto, A. Gadea, B. Guiot, A. Latina, P. Mason, G. Montagnoli, F. Scarlassara, M. Trotta, G. de Angelis, F. Della Vedova, E. Farnea, F. Haas, S. Lenzi, S. Lunardi, R. Marginean, R. Menegazzo, D. R. Napoli, M. Nespolo, I. V. Pokrovsky, F. Recchia, M. Romoli, M.-D. Salsac, N. Soic, and J. J. Valiente-Dobóbon, Phys. Rev. C 76, (2007) 024604

7. D. Montanari, E. Farnea, S. Leoni, G. Pollarolo, L. Corradi, G. Benzoni, A. Gadea, E. Fioretto, A. Latina, G. Montagnoli, F. Scarlassara, A.M. Stefanini, S. Szilner, Eur. Phys. J. A 47 (2011) 4.

8. A. Winther, Nucl. Phys. A572, (1994) 191; Nucl. Phys A594, (1995) 203

9. Nucl. Data Sheets 92, 783 (2001); 94, 1 (2001); 94, (2001) 429; National Nuclear Data Center, http://www.nndc.bnl.gov

10. E. Caurier, G. Martínez-Pinedo, F. Nowacki, A. Poves and A.P. Zuker, Rev. Mod. Phys. 77, (2005) 427

11. F. Nowacki and A. Poves, Phys. Rev. C 79, (2009) 014310

12. D. Mengoni, J.J. Valiente-Dobón, A. Gadea, S. Lunardi, S. M. Lenzi, R. Broda, A. Dewald, T. Pissulla, L. J. Angus, S. Aydin, D. Bazzacco, G. Benzoni, P. G. Bizzeti, A. M. Bizzeti-Sona, P. Boutachkov, L. Corradi, F. Crespi, G. de Angelis, E. Farnea, E. Fioretto, A. Goergen, M. Gorska, A. Gottardo, E. Grodner, A. M. Howard, W. Królas, S. Leoni, P. Mason, D. Montanari, G. Montagnoli, D. R. Napoli, A. Obertelli, R. Orlandi, T. Pawlat, G. Pollarolo, F. Recchia, A. Algora, B. Rubio, E. Sahin, F. Scarlassara, R. Silvestri, J. F. Smith, A. M. Stefanini, D. Steppenbeck, S. Szilner, C. A. Ur, P. T. Wady, and J. Wrzesiński, Phys. Rev. C 82, (2010) 024308

13. S. Bhattacharyya, M. Rejmund, A. Navin, E. Caurier, F. Nowacki, A. Poves, R. Chapman, D. O'Donnell, M. Gelin, A. Hodsdon, X. Liang, W. Mittig, G. Mukherjee, F. Rejmund, M. Rousseau, P. Roussel-Chomaz, K.M. Spohr, and Ch. Theisen, Phys. Rev. Lett. 101, (2008) 032501 